PROCEEDINGS OF THE

AMERICAN MATHEMATICAL SOCIETY

Volume 135, Number 6, June 2007, Pages 1865-1873

S 0002-9939(06)08675-8

Article electronically published on November 7, 2006

\title{
HANKEL OPERATORS WITH UNBOUNDED SYMBOLS
}

\author{
P. AHERN AND E. H. YOUSSFI
}

(Communicated by Joseph A. Ball)

\begin{abstract}
We prove that there are holomorphic functions $f$ in the Hardy space of the unit ball or the bidisc such that the big Hankel operator with symbol $\bar{f}$ is bounded and for any holomorphic function $g$ the function $\bar{f}+g$ cannot be bounded.
\end{abstract}

\section{INTRODUCTION}

Throughout this note $\Omega=\Omega_{n}$ will denote the domain in $\mathbb{C}^{n}$ which is either the unit ball $\mathbb{B}_{n}$ or the the unit polydisc $\mathbb{D}^{n}$, where $\mathbb{D}$ is the unit disc in $\mathbb{C}$. We let $\sigma$ denote the normalized Lebesgue measure on the Shilov boundary $\partial_{s} \Omega$ of $\Omega$. For the unit ball then $\partial_{s} \mathbb{B}_{n}:=\mathbb{S}$ is the unit sphere and for the unit polydisc, $\partial_{s} \mathbb{D}^{n}=\mathbb{T}^{n}$ where $\mathbb{T}$ is the unit circle. Let $H^{2}(\Omega)$ be the Hardy space on $\Omega$ with respect to $\sigma$. The Berezin transform of a function $f \in L^{2}(\sigma)$ is defined by

$$
\widetilde{f}(z):=\int_{\partial_{s} \Omega} f(\xi) P(z, \xi) d \sigma(\xi), z \in \Omega
$$

where $P(z, \xi)$ is the Poisson kernel of $\Omega$ with respect to $\sigma$. We follow Coburn [C] to define the space $\widetilde{B M O}(\partial \Omega)$ of functions with bounded mean oscillation with respect to $\sigma$ as the subspace of functions $f \in L^{2}(\sigma)$ that satisfy

$$
\sup _{z \in \Omega}\left[\widetilde{|f|^{2}}(z)-|\widetilde{f}|^{2}(z)\right]<\infty .
$$

We point out that in the one-dimensional case this definition gives the classical $B M O$ space. See [Z1]. The subspace of $\widetilde{B M O}(\partial \Omega)$ consisting of those analytic functions on $\Omega$ will be denoted by $\widetilde{B M O A}(\partial \Omega)$. In the case of the unit ball the space $\widetilde{B M O A}(\mathbb{B})$ was shown to be the dual of the Hardy space $H^{1}(\mathbb{B})$. See Theorem 5.13 of $\mathrm{Z2}$. For the polydisc case we show in Proposition 3.5 below that $\widetilde{B M O}$ coincides with the small bmo defined by Cotlar and Sadosky [CS]. It is not the dual of the real $H^{1}\left(\mathbb{T}^{2}\right)$, and its predual is given by Theorem 1.5 of $[\mathrm{CS}$.

The big Hankel operator $H_{f}$ with symbol $f \in L^{2}(\sigma)$ is defined by

$$
H_{f}(g):=(I-P)(f g), \quad g \in H^{\infty}(\Omega) .
$$

We recall that in the one variable setting $\Omega=\mathbb{D}$, if a function $f$ is in $H^{2}(\mathbb{D})$, then the big Hankel operator $H_{\bar{f}}$ is bounded on $H^{2}(\mathbb{T})$ if and only if there is another $g \in H^{2}(\mathbb{T})$ such that $\bar{f}+g$ is bounded. See [Z1]. The goal of this note is to show this

Received by the editors November 24, 2005 and, in revised form, February 16, 2006.

2000 Mathematics Subject Classification. Primary 47B35, 32A35, 32A25. 
is no longer true in higher dimensions. In particular, this answers in the negative a question by E. Strouse [S] which she raised for the case of the bidisc. We shall establish the following.

Theorem A. There are functions $f \in H^{2}\left(\Omega_{2}\right)$ such that the big Hankel operator $H_{\bar{f}}$ is bounded on $H^{2}\left(\Omega_{2}\right)$ but $\bar{f}+g$ is unbounded for any holomorphic function $g$ on $\Omega_{2}$.

We point out that the existence of big Hankel operators with not necessarily antianalytic unbounded symbols was estabished in [CS] and [BT] for the case of the bidisc.

It is not hard to see [C] that if $f \in L^{2}\left(\partial_{s} \Omega\right)$ and both operators $H_{f}$ and $H_{\bar{f}}$ are bounded, then $f \in \widetilde{B M O}\left(\partial_{s} \Omega\right)$. The converse is true for the bidisc. Indeed,

Theorem B. Let $f \in L^{2}\left(\mathbb{T}^{2}\right)$. Then $f \in \widetilde{B M O}\left(\mathbb{T}^{2}\right)$ if and only if the big Hankel operators $H_{f}$ and $H_{\bar{f}}$ are bounded on $H^{2}\left(\mathbb{D}^{2}\right)$.

The analogue of Theorem B for the unit ball in $\mathbb{C}^{2}$ remains open.

\section{The BALL CASE}

We recall that the Bergman distance $\beta(\zeta, z)$ between two points $\zeta$ and $z$ in the unit disc $\mathbb{D}$ in the complex plane is given by

$$
\beta(\zeta, z)=\frac{1}{2} \log \frac{1+\left|\varphi_{z}(\zeta)\right|}{1-\left|\varphi_{z}(\zeta)\right|}, \text { where } \varphi_{z}(\zeta):=\frac{\zeta-z}{1-\zeta \bar{z}} .
$$

Lemma 2.1. There is a positive constant $C$ independent of $n>0$ such that

$$
I_{n}(z):=\int_{\mathbb{D}}\left(1-|\zeta|^{2}\right)^{\frac{n}{2}} \frac{\beta(\zeta, z)}{|1-\bar{\zeta} z|^{n+2}} d A(\zeta) \leq \frac{C}{(n+1)^{\frac{3}{2}}}\left(1-|z|^{2}\right)^{-\frac{n}{2}}
$$

for all $z \in \mathbb{D}$.

Proof. Setting $\zeta=\varphi_{z}(w)$ we see that

$$
\begin{aligned}
I_{n}(z) & =\int_{\mathbb{D}} \beta(0, w) \frac{\left(1-|w|^{2}\right)^{\frac{n}{2}}}{|1-\bar{\zeta} w|^{2}} d A(w) \\
& =\frac{\left(1-|z|^{2}\right)^{-\frac{n}{2}}}{2} \int_{0}^{1} r \log \frac{1+r}{1-r}\left(1-r^{2}\right)^{\frac{n}{2}} \frac{1}{2 \pi} \int_{0}^{2 \pi} \frac{d \theta}{\left|1-r \bar{z} e^{i \theta}\right|^{2}} d r \\
& =\frac{\left(1-|z|^{2}\right)^{-\frac{n}{2}}}{2} \int_{0}^{1} r \frac{\left(1-r^{2}\right)^{\frac{n}{2}}}{1-r^{2}|z|^{2}} \log \frac{1+r}{1-r} d r \\
& \leq \frac{\left(1-|z|^{2}\right)^{-\frac{n}{2}}}{2} \int_{0}^{1} r\left(1-r^{2}\right)^{\frac{n}{2}-1} \log \frac{1+r}{1-r} d r \\
& =\frac{\left(1-|z|^{2}\right)^{-\frac{n}{2}}}{2}\left(\int_{0}^{1} r \log (1+r)\left(1-r^{2}\right)^{\frac{n}{2}-1} d r\right. \\
& \left.\quad+\int_{0}^{1} r\left(1-r^{2}\right)^{\frac{n}{2}-1} \log \frac{1}{1-r} d r\right) .
\end{aligned}
$$

Integration by parts and the change of variable $t=1-r$ give that the second integral in the last equality is equal to $4 n^{-2}$. To estimate the first integral in terms 
of $n$, we have

$$
\begin{aligned}
\int_{0}^{1} r \log (1+r)\left(1-r^{2}\right)^{\frac{n}{2}-1} d r & =\frac{1}{n} \int_{0}^{1} \frac{\left(1-r^{2}\right)^{\frac{n}{2}}}{1+r} d r \\
& \leq \frac{1}{2 n} \int_{0}^{1}\left(1-r^{2}\right)^{\frac{n}{2}} d r \\
& =\frac{1}{2 n} \int_{0}^{\frac{\pi}{2}}(\sin t)^{n} d t \leq \frac{C}{n^{\frac{3}{2}}}
\end{aligned}
$$

Let $\mathcal{B}(\mathbb{D})$ be the Bloch space of $\mathbb{D}$ and if $F \in \mathcal{B}(\mathbb{D})$, we extend $F$ to the ball $\mathbb{B}_{2}$ by setting $F(z, w):=F(z)$. Then we have

Lemma 2.2. If $F \in \mathcal{B}(\mathbb{D})$, then the big Hankel operator $H_{\bar{F}}$ is bounded on the Hardy space $H^{2}\left(\mathbb{B}_{2}\right)$.

We write each $g \in H^{2}(\mathbb{B})$ in the form $g(z, w)=\sum_{n} g_{n}(z) w^{n}$, where the functions $g_{n}$ are holomorphic in $\mathbb{D}$. In addition, we have the identity

$$
\int_{\mathbb{S}} g_{n}(z) w^{n} \overline{g_{m}(z) w^{m}} d \sigma(z, w)= \begin{cases}0, & n \neq m \\ \int_{\mathbb{D}}\left|g_{n}(z)\right|^{2}\left(1-|z|^{2}\right)^{n} d A(z), & n=m .\end{cases}
$$

Hence

$$
\|g\|_{L^{2}(\mathbb{S})}^{2}=\sum_{n} \int_{\mathbb{D}}\left|g_{n}(z)\right|^{2}\left(1-|z|^{2}\right)^{n} d A(z) .
$$

On the other hand, for each $n$, we have that

$$
\begin{aligned}
\left(P\left(\bar{F} g_{n}\right)(z, w)\right. & =\int_{\mathbb{S}} \frac{\bar{F}(\zeta) g_{n}(\zeta) \eta^{n}}{(1-\bar{\zeta} z-\bar{\eta} w)^{2}} d \sigma(\zeta, \eta) \\
& =\int_{\mathbb{D}} \frac{\bar{F}(\zeta) g_{n}(\zeta)}{(1-\bar{\zeta} z-\bar{\eta} w)^{2}} \int_{0}^{2 \pi} \frac{\left(1-|\zeta|^{2}\right)^{\frac{n}{2}} e^{i n \theta}}{\left(1-\bar{\zeta} z-\sqrt{1-|\zeta|^{2}} w e^{i \theta}\right)^{2}} \frac{d \theta}{2 \pi} d A(\zeta) \\
& =\int_{\mathbb{D}} \frac{\bar{F}(\zeta) g_{n}(\zeta)\left(1-|\zeta|^{2}\right)^{\frac{n}{2}}}{(1-\bar{\zeta} z)^{2}} \int_{0}^{2 \pi} \frac{e^{i n \theta}}{\left(1-\frac{\sqrt{1-|\zeta|^{2}} w}{1-\bar{\zeta} z} e^{-i \theta}\right)^{2}} \frac{d \theta}{2 \pi} d A(\zeta) \\
& =(n+1) w^{n} \int_{\mathbb{D}} \frac{\bar{F}(\zeta) g_{n}(\zeta)\left(1-|\zeta|^{2}\right)^{n}}{(1-\bar{\zeta} z)^{n+2}} d A(\zeta) w^{n} .
\end{aligned}
$$

Since $(n+1) \frac{\left(1-|\zeta|^{2}\right)^{n}}{(1-\zeta z)^{n+2}}$ reproduces holomorphic functions on the unit disc, it follows that

$$
\begin{aligned}
\left(H_{\bar{F}} g\right)(z, w) & =\sum_{n}(n+1) w^{n} \int_{\mathbb{D}} \frac{\bar{F}(z)-\bar{F}(\zeta)}{(1-\bar{\zeta} z)^{n+2}} g_{n}(\zeta)\left(1-|\zeta|^{2}\right)^{n} d A(\zeta) \\
& =\sum_{n} H_{n}(z) w^{n},
\end{aligned}
$$

where

$$
H_{n}(z):=(n+1) \int_{\mathbb{D}} \frac{\bar{F}(z)-\bar{F}(\zeta)}{(1-\bar{\zeta} z)^{n+2}} g_{n}(\zeta)\left(1-|\zeta|^{2}\right)^{n} d A(\zeta) .
$$

Since $F \in \mathcal{B}(\mathbb{D})$, we see that $|F(z)-F(\zeta)| \leq\|F\|_{\mathcal{B}(\mathbb{D})} \beta(z, \zeta)$. Hence

$$
\left|H_{n}(z)\right| \leq(n+1)\|F\|_{\mathcal{B}(\mathbb{D})} \int_{\mathbb{D}} \frac{\beta(z, \zeta)\left|g_{n}(\zeta)\right|}{|1-\bar{\zeta} z|^{n+2}}\left(1-|\zeta|^{2}\right)^{n} d A(\zeta)
$$


so that by Hölder's inequality and Lemma 2.1 we see that

$$
\begin{aligned}
\left|H_{n}(z)\right|^{2} & \leq(n+1)^{2}\|F\|_{\mathcal{B}(\mathbb{D})}^{2}\left(I_{n}(z)\right)^{2} \int_{\mathbb{D}} \frac{\beta(z, \zeta)\left|g_{n}(\zeta)\right|^{2}\left(1-|\zeta|^{2}\right)^{\frac{3 n}{2}}}{|1-\bar{\zeta} z|^{n+2}} d A(\zeta) \\
& \leq C \sqrt{n}\|F\|_{\mathcal{B}(\mathbb{D})}^{2}\left(1-|z|^{2}\right)^{-\frac{n}{2}} \int_{\mathbb{D}} \frac{\beta(z, \zeta)\left|g_{n}(\zeta)\right|^{2}\left(1-|\zeta|^{2}\right)^{\frac{n}{2}}}{|1-\bar{\zeta} z|^{n+2}}\left(1-|\zeta|^{2}\right)^{n} d A(\zeta) .
\end{aligned}
$$

Therefore, again applying Lemma 2.1, we have

$$
\begin{aligned}
& \int_{\mathbb{D}}\left|H_{n}(z)\right|^{2}\left(1-|z|^{2}\right)^{n} d A(\zeta) \\
& \leq 2 \sqrt{n}\|F\|_{\mathcal{B}(\mathbb{D})}^{2} \int_{\mathbb{D}}\left|g_{n}(\zeta)\right|^{2}\left(1-|\zeta|^{2}\right)^{\frac{3 n}{2}} \int_{\mathbb{D}} \frac{\beta(z, \zeta)\left(1-|z|^{2}\right)^{\frac{n}{2}}}{|1-\bar{\zeta} z|^{n+2}} d A(z) d A(\zeta) \\
& \leq 2 \frac{\|F\|_{\mathcal{B}(\mathbb{D})}^{2}}{n} \int_{\mathbb{D}}\left|g_{n}(\zeta)\right|^{2}\left(1-|\zeta|^{2}\right)^{n} d A(\zeta) .
\end{aligned}
$$

Thus

$$
\begin{aligned}
\left\|\left(H_{\bar{F}} g\right)\right\|_{H^{2}(\mathbb{B})}^{2} & =\sum_{n} \int_{\mathbb{D}}\left|H_{n}(z)\right|^{2}\left(1-|z|^{2}\right)^{n} d A(\zeta) \\
& \leq \sum_{n} \frac{\|F\|_{\mathcal{B}(\mathbb{D})}^{2}}{n} \int_{\mathbb{D}}\left|g_{n}(\zeta)\right|^{2}\left(1-|\zeta|^{2}\right)^{n} d A(\zeta) \\
& \leq\|F\|_{\mathcal{B}(\mathbb{D})}^{2}\|g\|_{H^{2}(\mathbb{B})}^{2} .
\end{aligned}
$$

Proof of Theorem A in the ball case $\Omega_{2}=\mathbb{B}_{2}$. Let $F \in \mathcal{B}(\mathbb{D}) \backslash B M O A(\mathbb{T})$ be extended to $\mathbb{B}_{2}$ as the function $(z, w) \mapsto F(z)$. By Lemma 2.2 the big Hankel operator $H_{\bar{F}}$ is bounded on the Hardy space $H^{2}\left(\mathbb{B}_{2}\right)$. Assume that there is a holomorphic function $g$ on $\mathbb{B}_{2}$ such that the function $G:=\bar{F}+g$ is bounded. Then the function $z \mapsto G(z, 0)$ is bounded on the unit disc and hence in $B M O(\mathbb{T})$. This shows that both functions $F$ and $z \mapsto g(z, 0)$ are in $B M O A(\mathbb{T})$. This contradicts the fact that $F \notin B M O A(\mathbb{T})$.

\section{THE BIDISC CASE}

Let $b m o\left(\mathbb{T}^{2}\right)$ be the subspace of functions $f \in L^{1}\left(\mathbb{T}^{2}\right)$ such that $f$ is in $B M O(\mathbb{T})$ in each variable and

$$
\sup _{z \in \mathbb{T}}\|f(z, \cdot)\|_{B M O}+\sup _{w \in \mathbb{T}}\|f(\cdot, w)\|_{B M O}<+\infty .
$$

In particular, if $f \in L^{1}\left(\mathbb{T}^{2}\right)$, then $f$ is in $b m o\left(\mathbb{T}^{2}\right)$ if and only if

$$
\sup _{z \in \mathbb{T}}\|M f(z, \cdot)\|_{\infty}+\sup _{w \in \mathbb{T}}\|M f(\cdot, w)\|_{\infty}<+\infty
$$

where

$$
(M \varphi)(\zeta):=\sqrt{\widetilde{|\varphi|^{2}}(\zeta)-|\widetilde{\varphi}|^{2}(\zeta)}, \zeta \in \mathbb{D}, \varphi \in L^{2}(\mathbb{T})
$$

The subspace of $b m o\left(\mathbb{T}^{2}\right)$ consisting of holmorphic functions on $\mathbb{D}^{2}$ will be denoted by $\operatorname{bmoa}\left(\mathbb{T}^{2}\right)$

Theorem 3.1. If $\varphi \in L^{\infty}(\mathbb{D})$, then

$$
(T \varphi)(z, w):=\int_{\mathbb{D}} \frac{\varphi(\zeta)}{(1-z \bar{\zeta})(1-w \bar{\zeta})} d A(\zeta), \quad(z, w) \in \mathbb{T}^{2},
$$


is in bmoa $\left(\mathbb{T}^{2}\right)$. In addition, the operator $T$ is bounded from $L^{\infty}(\mathbb{D})$ into bmoa $\left(\mathbb{T}^{2}\right)$.

To prove this theorem we need some auxilary results. The following lemma can be found in [J].

Lemma 3.2. There is a positive constant $C$ such that

$$
\int_{\mathbb{D}} \frac{d A(\zeta) d}{|1-z \bar{\zeta}|^{\alpha}|1-w \bar{\zeta}|^{\beta}} \leq \begin{cases}C, & \alpha+\beta<2, \\ C \log \frac{1}{|1-z \bar{w}|}, & \alpha+\beta=2, \\ \frac{C}{|1-z \bar{w}|^{\alpha+\beta-2}}, & \alpha+\beta>2,\end{cases}
$$

for all $z, w \in \mathbb{D}$.

Lemma 3.3. There is a positive constant $C$ such that

$$
I(z, w):=\int_{\mathbb{D} \times \mathbb{D}} \frac{d A(\zeta) d A(\eta)}{|1-z \bar{\zeta}||1-z \bar{\eta}||1-w \bar{\zeta}||1-w \bar{\eta}||1-\zeta \bar{\eta}|} \leq \frac{C}{|1-z \bar{w}|}
$$

for all $z, w \in \mathbb{D}$.

Proof. We observe that $I(z, w) \leq I_{1}(z, w)+I_{2}(z, w)+I_{3}(z, w)$, where

$$
\begin{aligned}
I_{j}(z, w):= & \int_{\mathbb{E}_{j}} \frac{d A(\zeta) d A(\eta)}{|1-z \bar{\zeta}||1-z \bar{\eta}||1-w \bar{\zeta}||1-w \bar{\eta}||1-\zeta \bar{\eta}|}, j=1,2,3, \\
& \mathbb{E}_{1}:=\left\{(\zeta, \eta) \in \mathbb{D} \times \mathbb{D}:|1-\zeta \bar{\eta}| \leq \frac{1}{2}|1-z \bar{\zeta}|\right\}, \\
& \mathbb{E}_{2}:=\left\{(\zeta, \eta) \in \mathbb{D} \times \mathbb{D}:|1-\zeta \bar{\eta}| \leq \frac{1}{2}|1-w \bar{\eta}|\right\}
\end{aligned}
$$

and $\mathbb{E}_{3}$ is the complement of $\mathbb{E}_{1} \cup \mathbb{E}_{2}$ in $\mathbb{D} \times \mathbb{D}$. By symmetry the integrals $I_{1}(z, w)$ and $I_{2}(z, w)$ can be estimated similarly. Since by Lemma 3.2

$$
\begin{aligned}
I_{3}(z, w) & \leq 2 \int_{\mathbb{D} \times \mathbb{D}} \frac{d A(\zeta) d A(\eta)}{|1-z \bar{\zeta}|^{\frac{3}{2}}|1-z \bar{\eta}||1-w \bar{\zeta}||1-w \bar{\eta}|^{\frac{3}{2}}} \\
& \leq \frac{C}{|1-z \bar{w}|},
\end{aligned}
$$

we only need to estimate $I_{1}(z, w)$. To do so, we write $I_{1}(z, w)=I_{11}(z, w)+I_{12}(z, w)$, where

$$
I_{1 j}(z, w):=\int_{\mathbb{E}_{1 j}} \frac{d A(\zeta) d A(\eta)}{|1-z \bar{\zeta}||1-z \bar{\eta}||1-w \bar{\zeta}||1-w \bar{\eta} \| 1-\zeta \bar{\eta}|}, j=1,2,
$$

$\mathbb{E}_{11}:=\left\{(\zeta, \eta) \in \mathbb{E}_{1}:|1-\zeta \bar{\eta}| \leq \frac{1}{2}|1-w \bar{\zeta}|\right\}$ and $\mathbb{E}_{12}$ is the complement of $\mathbb{E}_{11}$ in $\mathbb{E}_{1}$. Set $R(z, w, \zeta):=\min (|1-z \bar{\zeta}|,|1-w \bar{\zeta}|)$. Since on $\mathbb{E}_{11}$ we have $|1-z \bar{\zeta}| \approx|1-z \bar{\eta}|$ and $|1-w \bar{\zeta}| \approx|1-w \bar{\eta}|$, it follows that

$$
\begin{aligned}
I_{11}(z, w) & \approx \int_{\mathbb{E}_{11}} \frac{d A(\zeta) d A(\eta)}{|1-z \bar{\zeta}|^{2}|| 1-\left.w \bar{\zeta}\right|^{2}|1-\zeta \bar{\eta}|} \\
& \leq \int_{\mathbb{D}} \int_{\left\{\eta \in \mathbb{D}:|1-\zeta \bar{\eta}| \leq \frac{1}{2} R(z, w, \zeta)\right\}} \frac{d A(\eta)}{|1-\zeta \bar{\eta}|} \frac{d A(\zeta)}{|1-z \bar{\zeta}|^{2}|| 1-\left.w \bar{\zeta}\right|^{2}} \\
& \leq \int_{\mathbb{D}} \frac{R(z, w, \zeta)}{|1-z \bar{\zeta}|^{2}|| 1-\left.w \bar{\zeta}\right|^{2}} d A(\zeta) \\
& \leq \frac{C}{|1-z \bar{w}|},
\end{aligned}
$$


where the latter inequality holds in view of Lemma 3.2. It now remains to establish the estimate of $I_{12}(z, w)$. We write $I_{12}(z, w)=\sum_{j=1}^{3} I_{12 j}(z, w)$, where

$$
\begin{aligned}
I_{12 j}(z, w):= & \int_{\mathbb{E}_{12 j}} \frac{d A(\zeta) d A(\eta)}{|1-z \bar{\zeta}||1-z \bar{\eta}||1-w \bar{\zeta}||1-w \bar{\eta}||1-\zeta \bar{\eta}|}, j=1,2,3, \\
& \mathbb{E}_{121}:=\left\{(\zeta, \eta) \in \mathbb{E}_{12}:|1-w \bar{\eta}| \leq \frac{1}{2}|1-\zeta \eta|\right\}, \\
& \mathbb{E}_{122}:=\left\{(\zeta, \eta) \in \mathbb{E}_{12}:|1-\zeta \bar{\eta}| \leq \frac{1}{2}|1-w \bar{\eta}|\right\},
\end{aligned}
$$

and $\mathbb{E}_{123}$ is the complement of $\mathbb{E}_{121} \cup \mathbb{E}_{122}$ in $\mathbb{E}_{12}$. On $\mathbb{E}_{121}$ we have $|1-w \bar{\zeta}| \approx|1-\zeta \bar{\eta}|$ and $|1-w \bar{\eta}| \leq C R(z, w, \zeta)$. Hence, by Lemma 3.2 ,

$$
\begin{aligned}
I_{121}(z, w) & \approx \int_{\mathbb{D}} \int_{\{\eta \in \mathbb{D}:|1-w \bar{\eta}| \leq C R(z, w, \zeta)\}} \frac{A(\eta)}{|1-w \bar{\eta}|} \frac{d A(\zeta)}{|1-z \bar{\zeta}|^{2}|| 1-\left.w \bar{\zeta}\right|^{2}} \\
& \leq C \int_{\mathbb{D}} \frac{R(z, w, \zeta)}{|1-z \bar{\zeta}|^{2}|| 1-\left.w \bar{\zeta}\right|^{2}} d A(\zeta) \\
& \leq \frac{C}{|1-z \bar{w}|} .
\end{aligned}
$$

On $\mathbb{E}_{122}$ we have $|1-w \bar{\zeta}| \approx|1-w \bar{\eta}|$ and $|1-\zeta \bar{\eta}| \leq C R(z, w, \zeta)$. Hence as in the previous case we have

$$
\begin{aligned}
I_{122}(z, w) & \approx \int_{\mathbb{D}} \int_{\{\eta \in \mathbb{D}:|1-w \bar{\eta}| \leq C R(z, w, \zeta)\}} \frac{A(\eta)}{|1-w \bar{\eta}|} \frac{d A(\zeta)}{|1-z \bar{\zeta}|^{2}|| 1-\left.w \bar{\zeta}\right|^{2}} \\
& \leq \frac{C}{|1-z \bar{w}|} .
\end{aligned}
$$

Finally, on $\mathbb{E}_{123}$ we have $|1-w \bar{\eta}| \approx|1-\zeta \bar{\eta}|$ and $|1-w \bar{\zeta}| \leq 2|1-\zeta \bar{\eta}|$. Thus

$$
\begin{aligned}
I_{123}(z, w) & \approx \int_{\mathbb{D}} \int_{\left\{\eta \in \mathbb{D}:|1-\zeta \bar{\eta}| \leq \frac{1}{2}|1-z \bar{\zeta}|\right\}} \frac{A(\eta)}{|1-\zeta \bar{\eta}|^{\frac{3}{2}}} \frac{d A(\zeta)}{|1-z \bar{\zeta}|^{2}|| 1-\left.w \bar{\zeta}\right|^{\frac{3}{2}}} \\
& \leq C \int_{\mathbb{D}} \frac{d A(\zeta)}{|1-z \bar{\zeta}|^{\frac{3}{2}}|| 1-\left.w \bar{\zeta}\right|^{\frac{3}{2}}} \\
& \leq \frac{C}{|1-z \bar{w}|} .
\end{aligned}
$$

Proof of Theorem 3.1. Let

$$
f(z, w)=(T \varphi)(z, w)=\int_{\mathbb{D}} \frac{\varphi(\zeta)}{(1-z \bar{\zeta})(1-w \bar{\zeta})} d A(\zeta),(z, w) \in \mathbb{D}^{2} .
$$

Then $f$ is in the Hardy space $H^{2}\left(\mathbb{D}^{2}\right)$ and satisfies $\widetilde{f(\cdot, w)}(z)=f(z, w)$ and

$$
\begin{aligned}
|\widetilde{f(\cdot, w)}|^{2}(z) & =\frac{1}{2 i \pi} \int_{\mathbb{T}} \int_{\mathbb{D} \times \mathbb{D}} \frac{\varphi(\zeta) \overline{\varphi(\eta)}\left(1-|z|^{2}\right) d A(\zeta) d A(\eta)}{(1-\tau \bar{\zeta})(1-w \bar{\zeta})(1-\bar{\tau} \eta)(1-\bar{w} \eta)|1-z \bar{\tau}|^{2}} \frac{d \tau}{\tau} \\
& =\int_{\mathbb{D} \times \mathbb{D}} \frac{\varphi(\zeta) \overline{\varphi(\eta)}\left(1-\eta \bar{\zeta}|z|^{2}\right) d A(\zeta) d A(\eta)}{(1-z \bar{\zeta})(1-w \bar{\zeta})(1-\bar{z} \eta)(1-\bar{w} \eta)(1-\bar{\zeta} \eta)}
\end{aligned}
$$


by the Cauchy formula. Therefore, by Lemma 3.3 we see that

$$
\begin{aligned}
(M f(\cdot, w))^{2}(z) & =|\widetilde{f(\cdot, w)}|^{2}(z)-|\widetilde{f}(z, w)|^{2} \\
& =\left|\int_{\mathbb{D} \times \mathbb{D}} \frac{\zeta \bar{\eta} \varphi(\zeta) \overline{\varphi(\eta)}\left(1-|z|^{2}\right) d A(\zeta) d A(\eta)}{(1-z \bar{\zeta})(1-w \bar{\zeta})(1-\bar{z} \eta)(1-\bar{w} \eta)(1-\bar{\zeta} \eta)}\right| \\
& \leq\|\varphi\|_{\infty}^{2}\left(1-|z|^{2}\right) \int_{\mathbb{D} \times \mathbb{D}} \frac{d A(\zeta) d A(\eta)}{|(1-z \bar{\zeta})(1-w \bar{\zeta})(1-z \bar{\eta})(1-w \bar{\eta})(1-\zeta \bar{\eta})|} \\
& \leq C\|\varphi\|_{\infty}^{2} .
\end{aligned}
$$

Similarly we have $\|M f(z, \cdot)\|_{\infty} \leq C\|\varphi\|_{\infty}$, showing that $T \varphi \in \operatorname{bmoa}\left(\mathbb{T}^{2}\right)$ and

$$
\|T \varphi\|_{b m o a} \leq C\|\varphi\|_{\infty} \text {. }
$$

Theorem 3.4. Suppose that $h \in \mathcal{B}(\mathbb{D})$ and $h \notin B M O A(\mathbb{T})$ and let

$$
f(z, w):=\int_{\mathbb{D}} \frac{h(\zeta)}{(1-z \bar{\zeta})(1-w \bar{\zeta})} d A(\zeta),(z, w) \in \mathbb{T}^{2} .
$$

Then the big Hankel operator $H_{\bar{f}}$ is bounded on $H^{2}\left(\mathbb{D}^{2}\right)$, and for any holomorphic function $g$ on $\mathbb{D} \times \mathbb{D}$, the function $\bar{f}+g$ cannot be bounded.

Proof. Since $h \in \mathcal{B}(\mathbb{D})$, there is $\varphi \in L^{\infty}(\mathbb{D})$ such that

$$
h(\lambda)=\int_{\mathbb{D}} \frac{\varphi(\zeta)}{(1-\lambda \bar{\zeta})^{2}} d A(\zeta), \lambda \in \mathbb{D} .
$$

By the reproducing formula of the Bergman kernel and Theorem 3.1 we see that

$$
f(z, w):=\int_{\mathbb{D}} \frac{\varphi(\zeta)}{(1-z \bar{\zeta})(1-w \bar{\zeta})} d A(\zeta)=(T \varphi)(z, w) \in \operatorname{bmoa}\left(\mathbb{T}^{2}\right) .
$$

By [FS] we see that $H_{\bar{f}}$ is bounded on $H^{2}\left(\mathbb{D}^{2}\right)$. Now suppose that there is a holomorphic function $g$ on $\mathbb{D}^{2}$ such that the function $F:=\bar{f}+g$ is bounded. Then the restriction of $F$ to the diagonal is also bounded and hence in $B M O(\mathbb{T})$. Since if $\lambda \in \mathbb{D}$, we have $F(\lambda, \lambda)=\bar{h}(\lambda)+g(\lambda, \lambda)$, it follows that both functions $h$ and $g(\lambda, \lambda)$ are in $B M O A(\mathbb{T})$. This contradicts the fact that $h \notin B M O A(\mathbb{T})$.

Now, Theorem A in the bidisc case follows from Theorem 3.4.

Proposition 3.5. bmo $\left(\mathbb{T}^{2}\right)=\widetilde{B M O}\left(\mathbb{T}^{2}\right)$.

Proof. For $z, w \in \mathbb{D}$ and $\theta \in[0,2 \pi]$, set $P_{z}(\theta):=\frac{1-|z|^{2}}{\left|1-z e^{-i \theta}\right|^{2}}$ and define the measure $\mu_{z, w}$ on $[0,2 \pi] \times[0,2 \pi] \times[0,2 \pi] \times[0,2 \pi]$ by

$$
d \mu_{z, w}(\theta, s, \psi, t):=\frac{1}{(2 \pi)^{4}} P_{z}(\theta) P_{z}(\psi) P_{w}(s) P_{w}(t) d \theta d s d \psi d t .
$$

A little computing shows that if $f \in L^{2}\left(\mathbb{T}^{2}\right)$, then we have the following key equality:

$$
\begin{aligned}
& 2\left(\widetilde{|f|^{2}}-|\tilde{f}|^{2}\right)(z, w) \\
& \quad=\int_{0}^{2 \pi} \int_{0}^{2 \pi} \int_{0}^{2 \pi} \int_{0}^{2 \pi}\left|f\left(e^{i \theta}, e^{i s}\right)-f\left(e^{i \psi}, e^{i t}\right)\right|^{2} d \mu_{z, w}(\theta, s, \psi, t),
\end{aligned}
$$


which will be used to prove both inclusions. On the one hand, suppose that $f \in$ $b m o\left(\mathbb{T}^{2}\right)$. Then the key inequality and the triangle inequality imply that

$$
\sqrt{2\left(\widetilde{|f|^{2}}-|\widetilde{f}|^{2}\right)(z, w)} \leq \sqrt{A(z, w)}+\sqrt{B(z, w)}
$$

where

$$
\begin{aligned}
A(z, w) & :=\int_{0}^{2 \pi} \int_{0}^{2 \pi} \int_{0}^{2 \pi} \int_{0}^{2 \pi}\left|f\left(e^{i \theta}, e^{i s}\right)-f\left(e^{i \theta}, e^{i t}\right)\right|^{2} d \mu_{z, w}(\theta, s, \psi, t) \\
& =\frac{1}{(2 \pi)^{3}} \int_{0}^{2 \pi} \int_{0}^{2 \pi} \int_{0}^{2 \pi}\left|f\left(e^{i \theta}, e^{i s}\right)-f\left(e^{i \theta}, e^{i t}\right)\right|^{2} P_{z}(\theta) P_{w}(s) P_{w}(t) d \theta d s d t \\
& =\frac{1}{\pi} \int_{0}^{2 \pi}\left(M\left[f\left(e^{i \theta}, \cdot\right)\right](w)\right)^{2} P_{z}(\theta) d \theta
\end{aligned}
$$

and similarly

$$
\begin{aligned}
B(z, w) & :=\int_{0}^{2 \pi} \int_{0}^{2 \pi} \int_{0}^{2 \pi} \int_{0}^{2 \pi}\left|f\left(e^{i \theta}, e^{i t}\right)-f\left(e^{i \psi}, e^{i t}\right)\right|^{2} d \mu_{z, w}(\theta, s, \psi, t) \\
& =\frac{1}{\pi} \int_{0}^{2 \pi}\left(M\left[f\left(\cdot, e^{i t}\right)\right](z)\right)^{2} P_{w}(t), d t
\end{aligned}
$$

showing that $b m o\left(\mathbb{T}^{2}\right) \subseteq \widetilde{B M O}\left(\mathbb{T}^{2}\right)$.

On the other hand, suppose that $f \in \widetilde{B M O}\left(\mathbb{T}^{2}\right)$. By Proposition 1.3 of [CS], in order to show that $f \in b m o\left(\mathbb{T}^{2}\right)$, we only need prove that $f$ is of bounded mean oscillation on rectangles; that is, for some constant $C>0$ we have

$$
\frac{1}{|I|} \frac{1}{|J|} \int_{I \times J}\left|f\left(e^{i \theta}, e^{i s}\right)-f_{I \times J}\right|^{2} d \theta d s \leq C
$$

for all intervals $I, J \subset[0,2 \pi]$, where $|I|$ is the legnth of an interval $I$ and

$$
f_{I \times J}:=\frac{1}{|I||J|} \int_{I} \int_{J} f\left(e^{i \theta}, e^{i s}\right) d \theta d s .
$$

To do so, let $z=\sqrt{1-\varepsilon} e^{i \theta_{0}}$ and $w=\sqrt{1-\varepsilon} e^{i s_{0}}$ where $\theta_{0}, s_{0} \in[0,2 \pi]$ and $\varepsilon \in] 0,1]$, then for some constant $C>0$ independent of all parameters we have

$$
P_{z}(\theta) \geq \frac{C}{1-|z|^{2}} \quad \text { and } \quad P_{w}(\theta) \geq \frac{C}{1-|w|^{2}} .
$$

Thus by the key equality above we have

$$
\begin{aligned}
& 2\left(\widetilde{|f|^{2}}-|\widetilde{f}|^{2}\right)(z, w) \\
& \quad \geq \int_{\theta_{0}-\varepsilon}^{\theta_{0}+\varepsilon} \int_{s_{0}-\varepsilon}^{s_{0}+\varepsilon} \int_{\theta_{0}-\varepsilon}^{\theta_{0}+\varepsilon} \int_{s_{0}-\varepsilon}^{s_{0}+\varepsilon}\left|f\left(e^{i \theta}, e^{i s}\right)-f\left(e^{i \psi}, e^{i t}\right)\right|^{2} d \mu_{z, w}(\theta, s, \psi, t) \\
& \quad \geq \frac{C^{4}}{(2 \varepsilon \pi)^{4}} \int_{\theta_{0}-\varepsilon}^{\theta_{0}+\varepsilon} \int_{s_{0}-\varepsilon}^{s_{0}+\varepsilon} \int_{\theta_{0}-\varepsilon}^{\theta_{0}+\varepsilon} \int_{s_{0}-\varepsilon}^{s_{0}+\varepsilon}\left|f\left(e^{i \theta}, e^{i s}\right)-f\left(e^{i \psi}, e^{i t}\right)\right|^{2} d t d \psi d s d \theta \\
& \quad=\frac{2 C^{4}}{(2 \pi)^{4}} \frac{1}{(2 \varepsilon)^{2}} \int_{\theta_{0}-\varepsilon}^{\theta_{0}+\varepsilon} \int_{s_{0}-\varepsilon}^{s_{0}+\varepsilon}\left|f\left(e^{i \theta}, e^{i s}\right)-f_{\theta_{0}-\varepsilon, \theta_{0}+\varepsilon[\times] s_{0}-\varepsilon, s_{0}+\varepsilon \mid}\right|^{2} d s d \theta .
\end{aligned}
$$


This shows that

$$
\frac{1}{|I|} \frac{1}{|J|} \int_{I \times J}\left|f\left(e^{i \theta}, e^{i s}\right)-f_{I \times J}\right|^{2} d \theta d s \leq \frac{(2 \pi)^{4}}{C^{4}} \sup _{z, w \in \mathbb{D}}\left(\widetilde{|f|^{2}}-|\widetilde{f}|^{2}\right)(z, w)
$$

for all intervals $I, J$ with length less than or equal to 2. Similar argument shows that

$$
\sup _{|I| \leq 2,|J|>2} \frac{1}{|I|} \frac{1}{|J|} \int_{I \times J}\left|f\left(e^{i \theta}, e^{i s}\right)-f_{I \times J}\right|^{2} d \theta d s \leq \frac{(2 \pi)^{2}}{C^{2}} \sup _{z \in \mathbb{D}}\left(\widetilde{|f|^{2}}-|\tilde{f}|^{2}\right)(z, 0),
$$

and by symmetry we have

$$
\sup _{|J| \leq 2,|I|>2} \frac{1}{|I|} \frac{1}{|J|} \int_{I \times J}\left|f\left(e^{i \theta}, e^{i s}\right)-f_{I \times J}\right|^{2} d \theta d s \leq \frac{(2 \pi)^{2}}{C^{2}} \sup _{w \in \mathbb{D}}\left(\widetilde{|f|^{2}}-|\tilde{f}|^{2}\right)(0, w) .
$$

The case where both intervals have length greater than 2 can be handled easily.

Proposition 3.5, combined with Theorem 2.1 of [FS], proves Theorem B and answers in the affirmative a conjecture due to Berger, Coburn and Zhu [C] for the bidisc case.

\section{REFERENCES}

[BT] M. Bakonyi and D. Timotin, On a conjecture of Coltar and Sadosky on multidimensional Hankel operators, C. R. Acad. Sci. Paris t. 325, Série I (1997), 1071-1075. MR.1614007 (99b:47032)

[C] L. Coburn, Toeplitz operators, quantum mechanics and mean oscillation in the Bergman metric, Proceedings of Symposia in Pure Mathematics 51 (1990), 97-104. MR.1077380 $(92 \mathrm{j}: 47045)$

[CS] M. Cotlar and C. Sadosky, Two distinguished subspaces of product BMO and Nehari-AAK theory of Hankel operators on the torus, Integral Equations and Operator Theory 26 (1996), 273-304. MR 1415032(98e:47043)

[FS] S. Ferguson and C. Sadosky, Characterizations of bounded mean oscillation on the polydisc in terms of Hankel operators and Carleson measures, J. Anal. Math. 81 (2000), 239-267. MR1785283 (2001h:47040)

[J] S. Jackobson, The harmonic Bergman kernel and the Friedrichs operator, Ark. Mat. 40 (2002), 89-104. MR1948888(2003j:30019)

[S] E. Strouse, Conference in Holomorphic Function Spaces and their Operators, CIRM Marseille, 2002 .

[Z1] K. Zhu, Operator Theory in Function Spaces, Marcel Dekker, New York, 1990. MR.1074007 (92c:47031)

[Z2] K. Zhu, Spaces of holomorphic functions in the unit ball, Graduate Texts in Math., 226, Springer, New York, 2005. MR2115155 (2006d:46035)

Department of Mathematics, University of Wisconsin, 480 Lincoln Drive, Madison, WISCONSIN 53705

E-mail address: ahern@math.wisc.edu

LATP, U.M.R. C.N.R.S. 6632, CMi, Université de Provence, 39 Rue F-Joliot-Curie, 13453 Marseille Cedex 13, France

E-mail address: youssfi@gyptis.univ-mrs.fr 